\title{
MedienPädagogik
}

www. medienpaed.com

Zeitschrift für

Theorie und Praxis

der Medienbildung

ISSN 1424-3636

Themenheft Nr. 23: Visuelle Kompetenz

\section{Eine schrecklich nette Familie?! Sozialen Wandel bildkompetent untersuchen und gestalten}

Anna Maria Loffredo

\begin{abstract}
Zugänge zu Bildern erfordern bilddidaktische Überlegungen, die im Kunstunterricht ein zentrales Anliegen einnehmen, um subjektorientierte und kompetenzorientierte Lernprozesse zugleich für Schüler/innen zu ermöglichen. Die kunstpädagogischen Handlungsfelder beziehen sich jedoch nicht nur auf die rezeptivreflexive, sondern auch auf die gestaltende Herstellung von Bildern. Die Fotografie stellt dabei ein Medium und eine Bildart dar, die mittlerweile technisch schnell verfügbar, vermeintlich zügig erfassbar und selbst leicht umsetzbar erscheint. Bildnerisch-ästhetische Strukturen entstehen jedoch vor dem Hintergrund eines kulturellen Bildreservoirs, das historisch gewachsen ist und wofür sich Jugendliche die nötigen Kenntnisse und Fähigkeiten rezeptiver, produktiver und reflexiver Art erst aneignen müssen. Am Beispiel von fotografischen Gruppenporträts zum Thema «Familie» werden in Referenz zur Bildgattung in der Malerei in diesem Beitrag ein didaktisierter Zugang zur Verlangsamung der Bildwahrnehmung, eine bildspezifische Untersuchungsmethode in Form von vergleichenden Kompositionsskizzen und Gestaltungslösungen aus einem Leistungskurs der Jahrgangsstufe 12 (G9) vorgestellt.
\end{abstract}

\section{Fotografische Bilder im Kunstunterricht}

Im Zentrum des Kunstunterrichts steht das Bild. Der Begriff «Bild» bezieht sich hier fortlaufend auf alle gestalteten Produkte und Prozesse im Sinne eines «offenen» Bildbegriffs, den der Kunstpädagoge Rolf Niehoff vor dem Hintergrund der fachtheoretischen Entwicklung beispielhaft umreisst: «Bild umfasst gestaltete Objekte, Prozesse und Situationen, umfasst verschiedene Bildsorten - z. B. das Passbild, das Herrscherbildnis, das Starporträt -, wird durch verschiedene Medien erzeugt - z. B. mittels Malerei, Plastik, digitaler Fotografie -, stammt aus verschiedenen bildnerischen Gestaltungsbereichen - z. B. aus der Kunst, der Architektur, dem Design» (Niehoff 2009, 15, Hervorhebung im Original). Im aktuellen Fachdiskurs und darüber hinaus spricht man von einem «pictorial turn» bzw. «iconic turn», um die Bedeutsamkeit der Bilder zu benennen. ${ }^{1}$ In Anbetracht dessen wird die ge-

1 Der US-amerikanische Kulturwissenschaftler W. J. Thomas Mitchell bringt für die sich abzeichnende Kulturwende von der Sprache hin zur Ausweitung und Verfügbarkeit neuer Bildmedien den Begriff «pictorial turn» ein (Mitchell 1994, 3 ff). Der Kunstwissenschaftler Gottfried Boehm prägte dafür den 
genwärtige Bildkultur von historischen Bildern und ihren spezifischen Merkmalen gespeist. Als medial vermittelte Folie tragen Bilder massgeblich zu den Weltkonstruktionen der heutigen Kinder und Jugendlichen bei (Niehoff 2012, 109), wofür sie mit einem Repertoire an Wahrnehmungs-, Analyse-, Gestaltungs- und Reflexionsfähigkeiten ausgebildet werden müssen, das dem medialen, vor allem technisch rasanten Wandel mit seinen Herstellungs- und Verabeitungsbedingungen adäquat gewachsen bleibt. Neben technischen Fähigkeiten ist der Aufbau von - kritischen - Einstellungen im Sinne einer ästhetischen Urteilsfähigkeit ein Kernanliegen in der Kompetenzvermittlung durch Bilder.

«Bildkompetenz» ${ }^{2}$ und «Bildlesekompetenz» sind demnach Bausteine der «Kulturkompetenz», die als allgemeinbildender Auftrag aller schulischen Fächer angesehen wird. Laut dem Kunstdidaktiker Kunibert Bering tritt die Kunst als ein wesentliches Medium der Orientierung auf (Bering 2010, 287), um Kulturkompetenz im Sinne des kulturellen Gedächtnisses in seiner autopoietischen Funktion zu pflegen und im Kunstunterricht die Einheit von Erinnern und Vergessen zwecks zukunftsfähiger Anschlussfähigkeit in der Kommunikation zu üben. "Ohne Kenntnis und Reflexion seiner historischen Genese ist Kultur als fundamentales Zeichensystem zur Konstituierung menschlicher Gemeinschaften nicht denkbar» (Bering 2010, 286). Das ist der Terminologie «visual literacy» entlehnt, die eine Unzahl modifizierter Begriffsversionen nach sich zog und im Grunde «to make meaning from images, the «reader uses the critical skills of exploration, critique and reflection» (Bamford o. A., 1) meint. «Visual literacy is a gradual process of gaining greater sophistication of perception, conception, visual and linguistic vocabulary» (Bamford o. A., 4), woran man durch das Erlernen und Einüben der Grammatik, Syntax und Semantik, vergleichbar mit dem Erlernen eines sprachlichen Zeichensystems/Codierung, inhaltlich und methodisch fachkundig aufbereitet und kulturspezifisch herangeführt werden muss. Dies muss vorrangig von den sogenannten «Bild»-Experten, den Kunstpädagogen, geleistet werden, weil dem Unterrichtsfach Kunst explizit das Sichtbare und das Sichtbar Machen im Wesen obliegt (Sowa 2010, 161; Bering et al. 2006, 115).

In der aktuellen Omnipräsenz von Bildern, der viel zitierten (multimedialen) «Bilderflut», ist es auf medialer Ebene zwangsläufig nötig, zwischen traditionellen/ klassischen Bildarten der Bildenden Kunst (Zeichnung, Malerei, Druckgrafik, später dann auch Bildhauerei) und Bildern der sogenannten Neuen Medien (Fotografie, Video, Mixed-Media usw.) und der Alltagswelt verbindend vorzugehen, d.h. di-

inzwischen gebräuchlicheren Begriff «iconic turn» (Boehm 1994, 11 ff). Mit Blick auf die voraussichtliche Entwicklung, dass zunehmend 3D-Darstellungen die Bedeutsamkeit des Raumes für ein verändertes Bild vom Bild akzentuieren, wird auch der Begriff «spatial turn» gehandelt (Meyer u. Sabisch 2009, 13).

2 Für die genauere Bestimmung von «Bildkompetenz» benennt Rolf Niehoff die bildstrukturale, -inhaltliche, -geschichtliche, biografische, komparative und crossmediale Dimension (Niehoff 2009, $24 \mathrm{ff}$. 
daktische Brücken zwischen der Lebenswelt der Schüler und dem Bildungsauftrag von Kulturkompetenz zu schlagen.

Der Umgang mit Fotografie gilt lange noch nicht als selbstverständlicher Teil visueller Kompetenz in der Kunstunterrichtspraxis, was am Beispiel curricularer Vorgaben für das Bundesland Nordrhein-Westfalen veranschaulicht werden kann. So wurde seit Einführung des Zentralabiturs 2009 erst ein Jahr später Fotografie am Beispiel von Cindy Sherman und Gregory Crewdson verpflichtend gesetzt. Weitere vier Jahre danach wurde anstelle der durchgängig beibehaltenen amerikanischen Fotografin Cindy Sherman der deutsche Fotograf Andreas Gursky in die Obligatorik aufgenommen (http://www.standardsicherung.schulministerium.nrw. de/abitur/abitur-gymnasiale-oberstufe/, 26.12.2012). Über den gleichberechtigten Einsatz fotografischer Bilder im Kunstunterricht der Sekundarstufe I kann lediglich spekuliert werden.

Es gilt Qualifikationen durch «visuelle Kompetenz» und «Bildkompetenz» in gegenseitiger Ergänzung im Kunstunterricht zu vermitteln. "¿Visuelle Kompetenz» meint eher die rezeptive, d. h. die erlebnishafte, analysierende und deutende Auseinandersetzung mit visuellen Gestaltungen unter Einbeziehung der räumlichen und haptischen Erfahrung, während der Begriff (Bildkompetenz` auch den produktiv-gestalterischen Aspekt einbezieht» (Bering et al. 2006, 9). Beide Ansätze, sei es als Einbettung der «visuellen Kompetenz» in die «Kulturkompetenz» stellvertretend durch Kunibert Bering oder als «Bild(lese)kompetenz» stellvertretend durch Rolf Niehoff, bilden den didaktischen Ausgangspunkt für das im Folgenden durchgeführte Unterrichtsvorhaben im Fach Kunst.

\section{Staatliche Förderung ausserschulischer Kooperationen}

In Nordrhein-Westfalen gibt es für ausserschulische Kooperationen mit Künstlern im Kunstunterricht das Förderprogramm «Kultur \& Schule» (www.kulturundschule. de). Eine kritische Stellungnahme zu dem von der Landesregierung angebotenen Förderprogramm wird hier nicht vorgenommen, kann aber an anderer Stelle nachgelesen werden (Loffredo u. Zimmermann 2011, 139 ff). Zusammen mit dem Kölner Fotografen, Journalisten und Blogger Damian Zimmermann³ (www.damianzimmermann.de/blog) wurde 2009 das erste Mal eine gemeinsame Unterrichtsreihe zu fotografischen Gruppenporträts unter dem Titel «One for all and all for all» zu inszenierten Berufsgruppen erprobt. Das Kooperationsprojekt war Teil einer fotografischen Erstausstellung von Schülerarbeiten aus dem Kunstunterricht im Café

3 Aktuell arbeitet Damian Zimmermann an dem künstlerischen Projekt «Paare: Menschenbilder aus der Bundesrepublik Deutschland zu Beginn des 21. Jahrhunderts», für das er gemeinsam mit seiner Lebensgefährtin, der Diplom-Fotodesignerin Nadine Preiss, Paare in ganz Deutschland porträtiert. Zuletzt wurden ihre Paare in DER SPIEGEL zu einem Artikel über das - fehlende - Eheglück (Bredow 2011, S. 126ff) sowie in der vierstündigen Dokumentation «Das Geheimnis der Liebe - Was Paare zusammenhält» auf VOX 2012 gezeigt (www.paareprojekt.de). 
Bauturm in Köln und fand im Rahmen der Internationalen Photoszene Köln (IPK), des Deutschen Jugendfotopreises 2010 und der Photokina im September/Oktober 2010 statt. Die im Folgenden beschriebenen Arbeitsergebnisse stellen eine Variation dar und entstanden zum Thema «Familie» in einem Leistungskurs der Jahrgangsstufe 12 an einem Gymnasium im Frühjahr 2012.

\section{Familie - ein heikles Thema im Kunstunterricht}

Bereits bei der ersten Zusammenarbeit 2009 wurde der thematische Rahmen «Familie» angedacht, als es darum ging, eine subjekt- und kompetenzorientierte Aufgabe zugleich im kunstpädagogischen Handlungsfeld der Produktion für die Schüler/innen zu entwickeln. Aus der Unsicherheit heraus, ob und wie man «Familie» zum Thema im Unterricht machen könne und wie die gesamte Unterrichtsreihe zu fotografischen Gruppenporträts überhaupt mit einem «fremden» Lehrer bei den Jugendlichen ankäme, wurde die Themenwahl der Berufsgruppen vorgezogen. Es entsteht sonst zu schnell die Gefahr, Schüler/innen damit zu überfordern, ein ggf. sehr persönliches Thema im Kunstunterricht verbildlichen zu müssen. Einerseits ist Familie Privatsache, die quasi hinter den eigenen vier Wänden gewahrt wird; andererseits ist sie eine universelle, d. h. für alle Kulturen prägende Sozialisationsinstanz, die in Deutschland zudem unter besonderem Schutz der staatlichen Ordnung im Grundgesetz steht (vgl. Art. 6, §1, http://www.bundestag.de/dokumente /rechtsgrundlagen/grundgesetz/gg_01.html, 24.3.2012). Als Definitionskriterien der Kern- oder Klein-Familie gelten in Deutschland «eine zumindest auf Dauer angelegte und legitimierte - Beziehung zwischen Mann und Frau, die gemeinsame Haushaltsführung und das Vorhandensein mindestens eines Kindes. Erst die Filiationsbeziehung, also das Kindschaftsverhältnis, macht aus einer Ehe eine F[amilie, eig. Anm.]» (Schäfers 2003, 81, Hervorhebung im Original). Die Merkmale von Familie sind zwar normativ geregelt, bieten sich aber in ihrer sozialen Praxis bisweilen anachronistisch zur ihrem Ideal in einem sozialen Wandel familialer Lebensformen milieuspezifisch dar.

Ein didaktisches Mass an Nähe und Distanz zur eigenen Familienbiografie muss bei den Unterrichtsangeboten für die Lernenden kalkuliert werden. Somit wird ein Zugang bedacht, der die privaten Interessen der Schüler/innen wahrt und zugleich einen kritischen Blick auf familiäre Lagen richtet und diese zu reflektieren vermag. Deshalb wurden für die eigene bildnerisch-ästhetische Tätigkeit die Bedingungen gestellt, sich selbst mit anderen Personen als Familie zu inszenieren. Dabei konnten die Schüler/innen entscheiden, inwiefern sie die eigenen Familienmitglieder oder gecastete Komparsen dafür einsetzen wollten. Das bedeutet, dass auch bei der Teilnahme eigener Angehöriger die Aufgabe einer Inszenierung im Mittelpunkt stand und keine sozial-kritisch dokumentarische, autobiografische Funktion ihrer privaten Verhältnisse gewünscht war. Dadurch entstanden ganz unterschied- 
liche Gruppen. Zwei Schülerinnen nahmen ihre eigenen Familien als «Personenmaterial», alle weiteren bildeten miteinander und mit weiteren Komparsen Gruppen, zu denen teilweise auch Hunde als sogenannte Familienmitglieder gehörten. Für die Lerngruppe des Leistungskurses ist Fotografie, insbesondere die inszenierte Fotografie nicht fremd, weil sie bereits in der Jahrgangsstufe 11 mit Zeichnung, Grafik, inszenierter Fotografie und Malerei mit der Bandbreite bildnerischer Ausdrucksformen vertraut gemacht wurden. Zudem war sie mit fachspezifischen Grundlagen und Vertiefungen inhaltlicher, methodischer und medialer Art vertraut, auf die der ausserschulische Kooperationspartner aufbauen konnte.

\section{Zur Auswahl fotografischer Impulse}

Wenn es um Gruppenbildnisse bzw. fotografische Familienbilder geht, kommt man bei der Unterrichtsplanung nicht an der Bildreihe Thomas Struths und der damit verbundenen Frage nach bildnerischen Gestaltungen als Spiegel und Reflexion gesellschaftlicher Normen und Vorstellungen vorbei. Die Reihe sollte jedoch ungern mit Struths Blick auf Familiendynamiken eröffnet werden, weil keinesfalls eine mimetische Lenkung der Schüler/innen gewünscht war.

An dieser Stelle wird ein wesentlicher Vorteil der Kooperation zwischen einer bezogen auf bildnerische Gestaltungsformen - eher generalistisch ausgebildeten Kunstpädagogin und einem spezialisierten Experten für Fotografie deutlich. Ein ausserschulischer Bildexperte für Fotografie verfügt natürlich über einen präziseren und auch breiteren Kenntnisstand dazu, welches fotografische Werk bzw. welche Einzelfotografie als Einstieg ausgewählt werden kann. Kunstpädagogen/innen) müssen in allen bildnerisch-ästhetischen Praxisfeldern hingegen universalkompetent auftreten und von Zeichnung bis Film alles im Unterricht lehren können.

\section{Folgende aktuelle Fotografen wurden für den inhaltlichen Kontext der Reihe ausgewählt:}

Annie Leibovitz (*1949)

Die US-amerikanische Fotografin Annie Leibovitz gehört zu den bekanntesten und bestbezahlten Fotografen der Welt. Mit ihren aufwendig inszenierten Porträts vieler US-Prominenter aus Politik, Wirtschaft und Kultur wurde sie weltberühmt. Zu ihren populärsten Arbeiten zählt das Doppelporträt, auf dem sich der nackte John Lennon an die bekleidete Yoko Ono klammert und das nur fünf Stunden vor Lennons Tod am 8. Dezember 1980 entstand. Ausserdem löste ihr Bild der hochschwangeren nackten Demi Moore 1991 einen Skandal in den USA aus. Zuletzt porträtierte sie 2009 die US-amerikanische Präsidentenfamilie Barack Obamas für die Vanity Fair und die Präsidial-Website. 
Thomas Struth (*1954)

Thomas Struth ist neben Andreas Gursky und Thomas Ruff einer der international erfolgreichsten Schüler von Bernd (und Hilla) Becher an der Kunstakademie Düsseldorf. Struth arbeitet in aufeinander folgenden Werkgruppen - zu den bekanntesten zählen seine Strassenansichten «Die Architektur der Strasse» im Sinne der «New Topographic Movement» sowie seine Familienporträts. In jedem seiner Familienbilder gibt Struth der Gruppe eine einzigartige Gestalt, die jedoch nur den gegenwärtigen Augenblick, in dem sich die Geschichten und Einzelschicksale der Familienmitglieder verbinden, darstellt.

Katharina Mayer (*1958)

Die in Düsseldorf lebende Katharina Mayer hat ihr Kunstgeschichtsstudium in Freiburg mit der Arbeit «Die surrealen Elemente im fotografischen Werk von Man Ray» abgeschlossen, bevor sie bei Bernd Becher in Düsseldorf studierte. Bekannt ist sie vor allem für ihre Familienbilder, die zwar nicht minder inszeniert sind als bei Thomas Struth, die aber durch aussergewöhnliche Positionierungen der Figuren sowie durch deren Körperhaltungen, Mimik und Kleidung häufig wie eine Bühneninszenierung und fast surreal wirken. Ihre Bilder entstehen oft, aber nicht ausschliesslich, als Auftragsarbeiten, die sie nach Absprache mit den Familien für ihr eigenes künstlerisches Projekt nutzt.

\section{Einstieg}

Um direkt und unvermittelt in das Projektthema einzusteigen, wurde den Schülern eine Fotografie Annie Leibovitz' aus dem Jahr 2001 präsentiert. Sie zeigt den USamerikanischen Präsidenten George W. Bush zusammen mit seinen engsten Vertrauten und Ministern: Colin Powell, Dick Cheney, Condoleezza Rice und Donald Rumsfeld sowie zwei weiteren, eher unbekannten Mitarbeitern. Allerdings wurden die Gesichter der Personen für den ersten Bildzugang durch weisse, nummerierte Kreise unkenntlich gemacht (Abb. 1). Damit sollte verdeutlicht werden, dass Körpersprache und die Anordnung der Figuren zueinander, die daraus resultierende Bildgestaltung sowie die Lichtsetzung bereits viel über die Rangordnung und die Gruppendynamik verraten, ohne dass die Schüler von den bekannten Gesichtern der Abgebildeten beeinflusst werden. Die Schüler wurden gefragt, welche Personen im Mittelpunkt der Bildaussage stehen, welche als Aussenseiter und wer eher als «unwichtiges Beiwerk» betrachtet werden kann. Es entwickelte sich eine kurze Diskussion, doch die Gruppe einigte sich bald auf Kernfiguren des Bildes. 


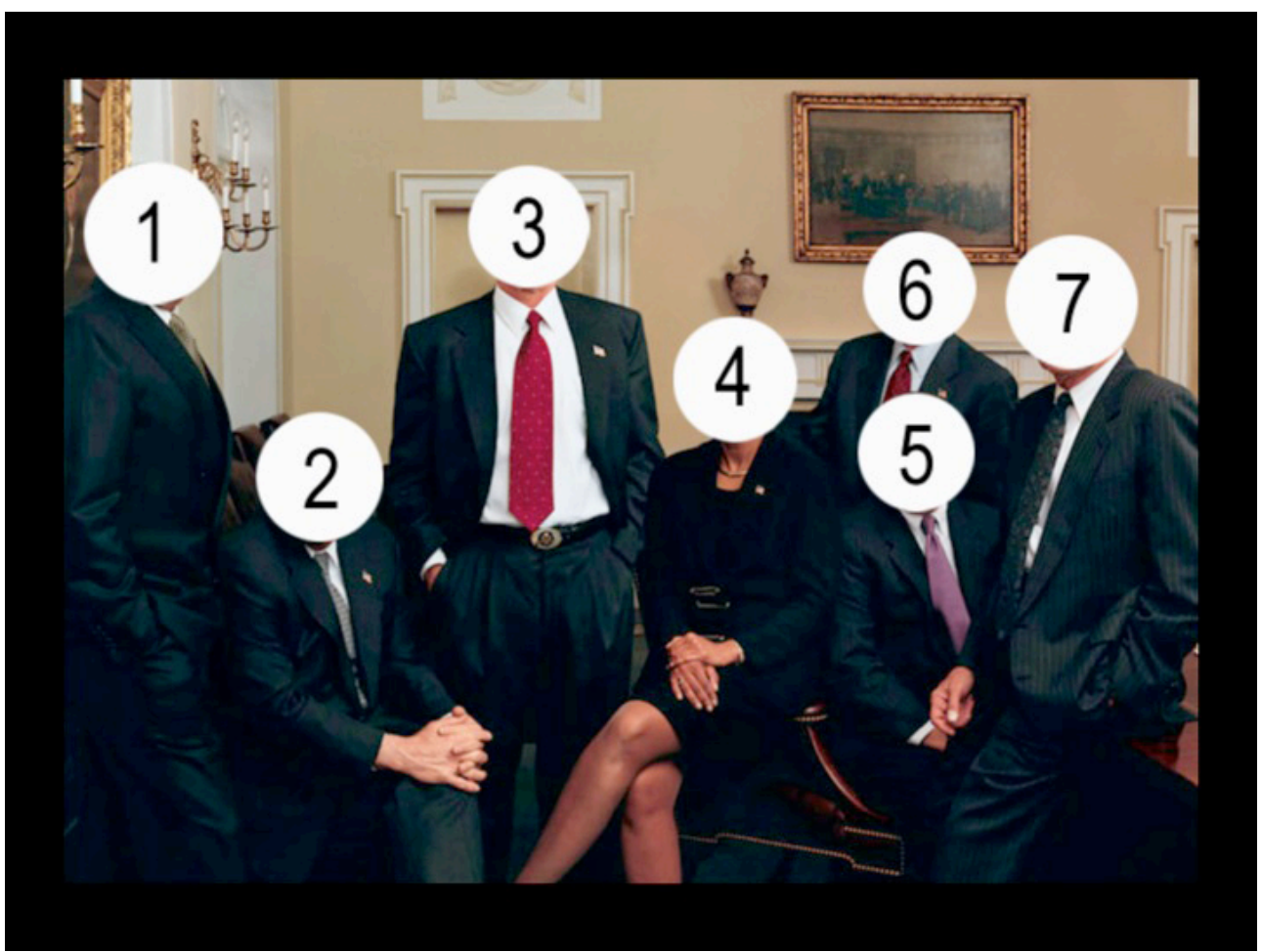

Abb. 1: In didaktischer Absicht bearbeitete Fotografie von Annie Leibovitz (Original siehe Abb.2)

Charakteristisch ist beispielsweise die metaphorische Sonderstellung des Präsidenten, der von Leibovitz innerhalb des sich im Hintergrund befindlichen Türrahmens gestellt wurde. Ausserdem sind den Schülern fast cowboyartige Attribute wie die hervorstechende goldene Gürtelschnalle und die betont lässige Körperhaltung frontal zum Betrachter mit den Händen in den Hosentaschen aufgefallen. Dass Aussenminister Colin Powell als «Schwarzes Schaf» des Kriegskabinetts später sein Amt niederlegen sollte, wurde von Leibovitz zu diesem Zeitpunkt wohl bereits geahnt - sie quetschte ihn wie einen unliebsamen Praktikanten an den linken Bildrand. Condoleezza Rice, die Powell als Aussenministerin folgen sollte, war zu diesem Zeitpunkt «nur» als Nationale Sicherheitsberaterin tätig - aber ihre strenge Körperhaltung und ihre prominente Platzierung direkt neben dem Präsidenten deuten an, wer im Kabinett tatsächlich das Sagen zu haben scheint. Spannend war auch die Bemerkung einer Schülerin, dass der an den Bildrand gedrückte Aussenseiter Colin Powell die einzige Person in der Fotografie ist, die mit Präsident Bush auf Augenhöhe agiert.

Im Anschluss wurden die anonymisierenden Kreise entfernt (Abb. 2) und die Gruppe bekam ihre zuvor aufgestellten Vermutungen grösstenteils bestätigt. Die mi- 
nimale Didaktisierung durch die nummerierten Kreise erleichtert in der ersten Bildannäherung einen spielerischen Ratetrieb der Schüler/innen, sodass der Aufmerksamkeitsfokus für die anstehende gestalterische Aufgabe sofort gegeben ist. Die Schülerbeteiligung am Unterrichtsgespräch erhöht sich potenziell. Die Flüchtigkeit in der Bildrezeption wird verlangsamt und intensiviert.

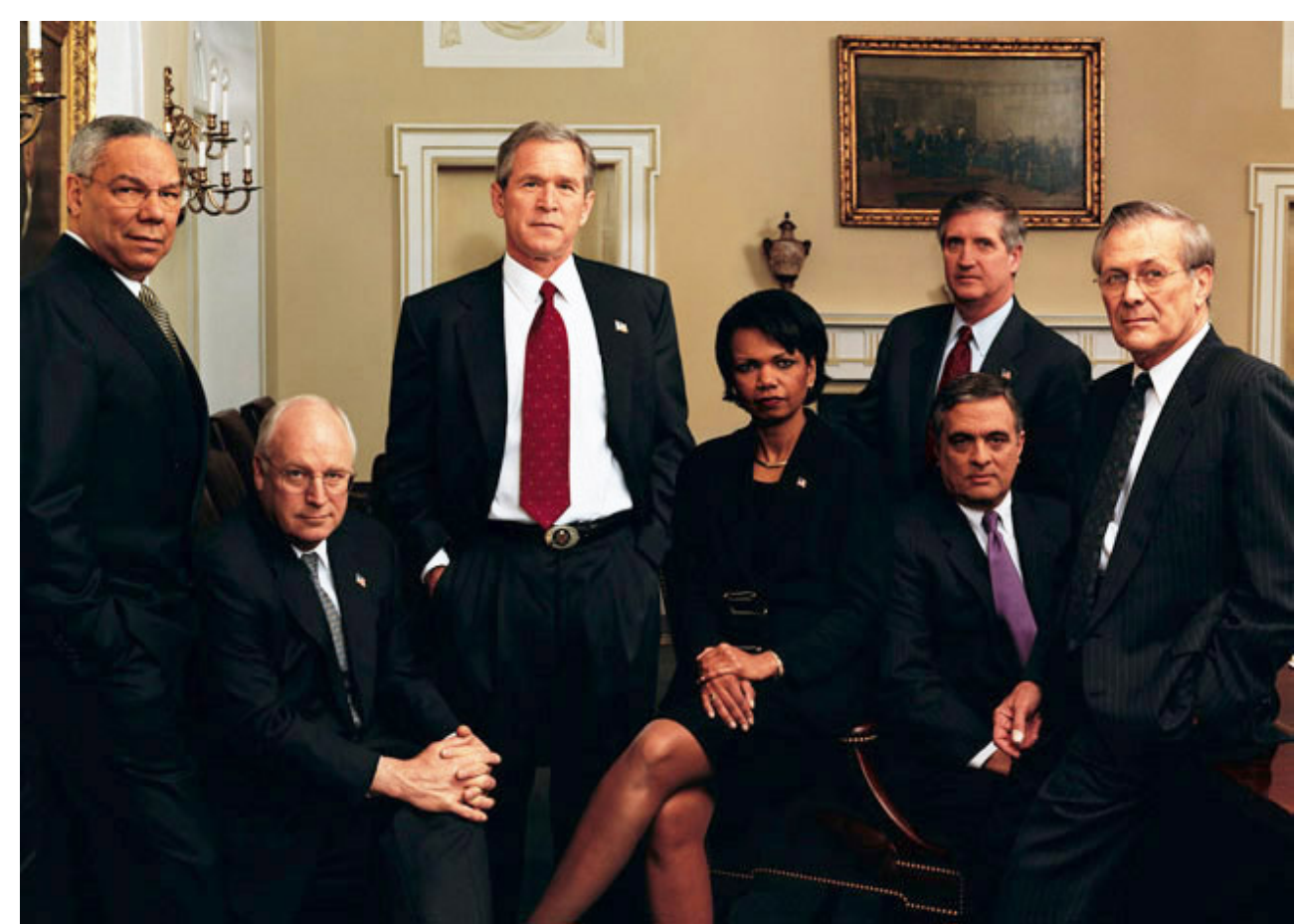

Abb. 2: Annie Leibovitz. George Bush und sein Kabinett im Cabinet Room, Dezember 2001

\section{Gestalterische Aufgabe}

Die gestalterische Aufgabe lautete wie folgt:

Ihre Aufgabe ist es, sich mit drei bis fünf weiteren Personen [Mitschülern/-innen oder Freunden/Bekannten/Verwandten] in einem fotografischen Gruppenporträt als eine fiktive Familie zu inszenieren. Dabei soll das hierarchische, ggf. auch emotionale Gefüge der Personen aus dem Zusammenspiel von Komposition, Lichtsetzung, Styling und Location auf den Punkt gebracht werden, um einen bestimmten Typus von Familie zu repräsentieren. Bedenken Sie dabei eine authentische Wiedergabe des sozialen Milieus (Bildung, Einkommen, Alter, Geschlecht, Ethnie usw.) der inszenierten Familie.

Erstellen Sie einen Plan zu folgenden Punkten: Zeitplan, bei Kooperation mit Mitschülern/-innen: Aufgabenverteilung und Übernahme von Verant- 
wortung, Location, Licht, Requisiten, Garderobe, Make-up, Hairstyling, Korrektur durch einen Fremdbetrachter.

Zwischenergebnisse sind zu den Beratungsterminen verpflichtend, müssen in ausgedruckter Form (A4) in guter Qualität vorliegen und werden bei der Schlussbeurteilung berücksichtigt.

Beratungstermin / Zwischenabgabe: ...

Abgabetermin: ...

Sobald die Schüler/innen die an sie gestellte Aufgabe erhalten, sollte man es als Lehrperson unterlassen, direkt danach noch weitere Informationen, Regeln, Bedingungen, Hinweise o. Ä. geben zu wollen. Denn die Teilnehmer/innen beginnen sofort mit einer kreativen Phase der Ideenfindung. Sie entwerfen und verwerfen, ggf. bis eine kleine Auswahl an möglichen Gestaltungskonzepten zumindest grob umrissen wurde. Die Aufgabe entwickelt ab diesem Zeitpunkt eine motivierende Dynamik, bei der die Lehrpersonen (gemeint sind hier sowohl die Kunstpädagogin als auch der ausserschulische Experte für Fotografie) lediglich bei Bedarf beratend von den Schülern miteinbezogen werden. Hier erweist sich ein "Team-Teaching» ${ }^{4}$ als gewinnbringender Vorteil im Unterrichtsgeschehen, da die Gruppen eine höhere Feedback-Frequenz von aussen erhalten können, wenn sie Hilfestellungen und Rückversicherungen benötigen. Die Lehrenden müssen sich nicht zerreissen, um der Lerngruppe gerecht zu werden. Dafür muss allerdings zwischen den als Lehrer fungierenden Kooperationspartnern sicher gestellt werden, dass sie als eingespieltes Team auftreten, ihre Stärken gegenseitig zu schätzen wissen, spezielle Fragen auch an den anderen vertrauensvoll delegieren können und keine gegensätzlichen Aussagen hinsichtlich der dem Unterricht zugrunde liegenden Intentionen machen. Erst dann macht eine arbeitsteilige Lernbegleitung im Team Sinn. In den gestellten Anforderungen wird darüber hinaus deutlich, dass bei den Schülern/-innen bereits Kenntnisse einer erfolgreichen Gruppenarbeit vorausgesetzt werden können, weil die Lehr-Lern-Kultur im Kunstunterricht seit der Jahrgangsstufe 11 auf Ansätzen des kooperativen Lernens von Gene Stanford, Ruth C. Cohn oder auch Norm Green phasenweise beruht. Ebenso kann der ausserschulische Kooperationspartner auf vorhandene Regeln des Miteinanders vertrauen, sodass er weitestgehend von Disziplinierungs- und Erziehungsmassnahmen in der Institution Schule entbunden wird, für die er in der Regel auch nicht ausgebildet wurde.

4 Die Psychologin Gesine Götting verweist z. B. auf die Helene-Lange-Schule in Wiesbaden, die in der PISA-Studie positiv abgeschnitten hatte und die u. a. mit der systematischen Umstellung des Unterrichts auf Team-Teaching eine von den Lehrkräften subjektiv grösser erlebte Berufszufriedenheit verzeichnen konnte (Götting 2007, 104). 


\section{Begleitimpulse und -übungen}

Das hierarchische Gefüge mehrerer Personen stellt eine formale Herausforderung dar, das zusätzlich mit den inhaltlich intendierten Bezügen der Bildlösung korrespondieren soll. Dafür eignen sich Kompositionsübungen an Beispielen aus dem historischen und aktuellen Bilderfundus unserer Kultur, damit eine Form-Wirkungskette schrittweise im Sinne der visuellen Kompetenz studiert werden kann. Die Erkenntnisse fliessen dann wieder in die eigenen Gestaltungsentscheidungen ein. In Kompositionsskizzen lassen sich u. a. die Form- und Flächenverteilung, der räumliche Aufbau, Farb- und/oder Hell-Dunkel-Unterschiede, die Blickführung und dominierende Bildelemente separieren und anschliessend miteinander in Bezug setzen.

Was man unter einer Familie versteht, ist in unserer Gesellschaft stark von dem bürgerlichen Ideal einer «Vater-Mutter-Kind-Beziehung» geprägt. Die Beziehung zwischen den einzelnen Familienmitgliedern wird in der Regel als harmonisch und aufeinander bezogen gedacht. Diese scheinbar unberührte Vorstellung durchdringt das tägliche Leben vieler Jugendlicher und wird in Bildern der Werbung, in Märchen, in Kinderserien usw. medial reproduziert. Die Beobachtungen, die die Schüler/innen selbst zu Hause machen, stimmen nur noch selten mit den Idealvorstellungen von Familie überein. Das gesellschaftliche Phänomen zwischen Sollund Ist-Zustand, welche Konstellationen in Familien herrschen (sollen), kann im Kunstunterricht anhand zweier im Kontrast zueinander stehenden Bildbeispiele untersucht werden. Der crossmediale Ansatz am Beispiel von Caspar David Friedrichs Gemälde «Die Lebensstufen», um 1834, und der Farbfotografie von Martin Parr aus «Seebad New Brighton Liverpool», 1983, führt den sozialen Wandel von Familie und die bildgeschichtliche Wechselbeziehung von Malerei und Fotografie zusammen. Dafür erhalten die Schüler/innen ein Übungsblatt mit vier Leitimpulsen, worauf sie bei der bildstrukturalen Untersuchung ihr Augenmerk richten sollen. Zu Beginn des Kompositionsvergleichs sollten keine Bilddaten angegeben werden, damit die Schüler/innen eine ungefähre Stileinordnung anhand kompositorischer Merkmale und bildnerischer Mittel vornehmen können (Abb. 3). Grundsätzlich ist es möglich, auf Kompositionsschemata bei der Bestimmung dominierender Bildachsen, Farb- und Formverteilungen usw. im Bildformat zurückzugreifen, z. B. der Goldene Schnitt, Isokephalie, Figura piramidale, Figura serpentinata (Hahne 2006, $14 \mathrm{f}$ ). Der Kern visueller Kompetenz besteht bei der Spezifizierung eines Bildaufbaus jedoch genau darin, auf den faktischen Bildbestand reagieren und ebenso ein vom Schema abweichendes Form-Inhalt-Gefüge ableiten zu können. «Es kann nicht Aufgabe des Kunstunterrichts sein, eine Geometrieaufgabe zu lösen» (Adolf Hölzel, in Wick 2012, 29). 


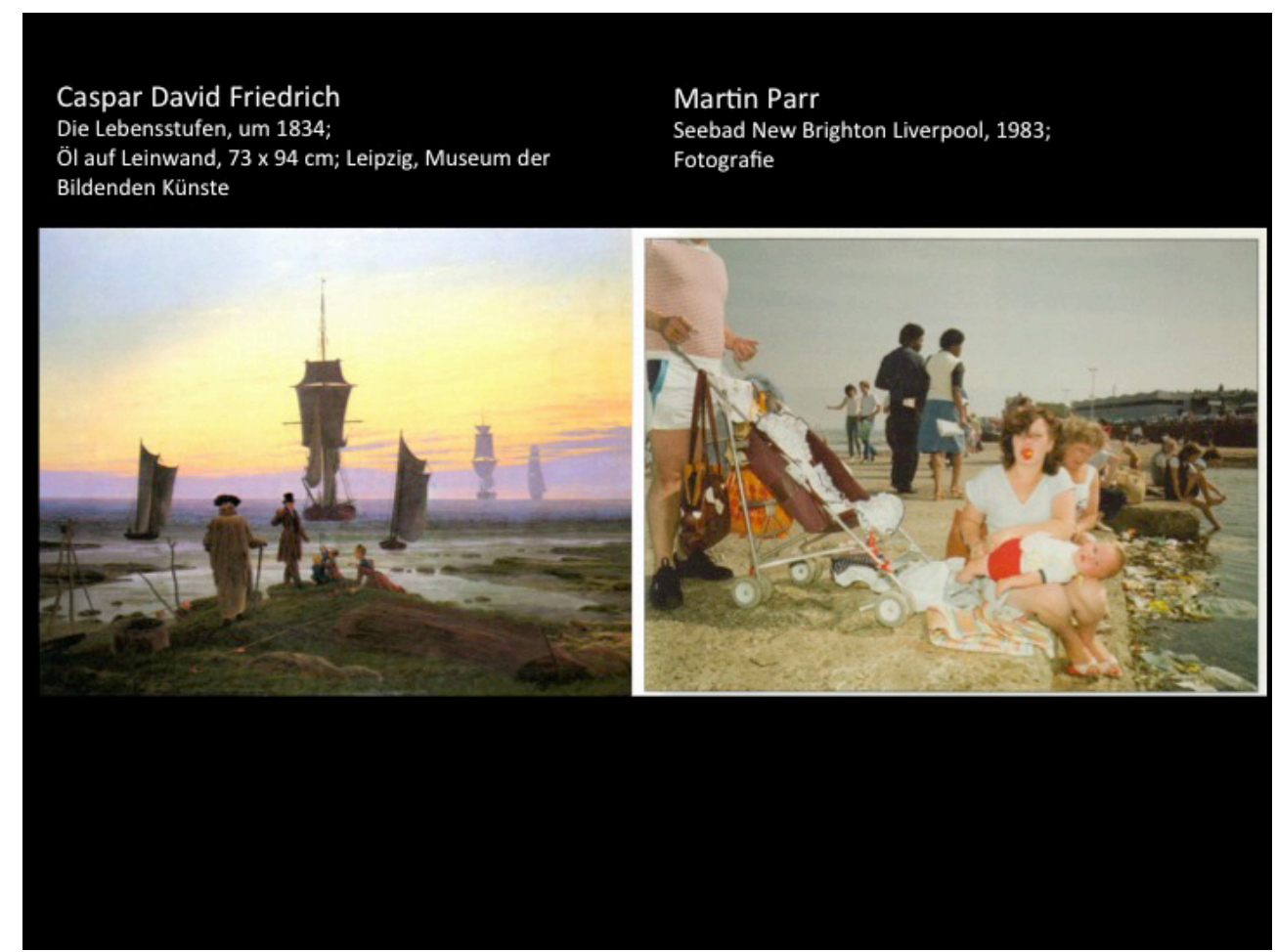

Abb. 3: Links: Caspar David Friedrich, «Die Lebensstufen», um 1834; rechts: Martin Parr, «Seebad New Brighton Liverpool», 1983

Die Fotografie von Parr steht im formalen Kontrast zum harmonisch-symmetrischen Bildaufbau bei Friedrich (Abb. 4, Abb. 5). Während die Anzahl und Grösse der Schiffe mit den Familienmitgliedern bei Friedrich korrespondieren, ist die Familienkonstellation vornehmlich auf Vater, Mutter und Kleinkind bei Parr beschränkt, wobei die ältere weibliche Person hinter der Mutter vielleicht die Oma sein könnte. Gewöhnlich zeichnet sich ein Familienporträt dadurch aus, dass die darauf abgebildeten Personen vollständig oder zumindest mit ihrem Gesicht deutlich zu erkennen sind. Hier wurde dem Vater sozusagen der Kopf abgeschnitten. Die Mutter und das Baby werden in einer unvorteilhaften Situation erwischt. Das Baby hängt schräg im Schoss und die Mutter hat alle Hände voll zu tun und klemmt sich daher den Schnuller in den Mund. Entgegen der romantischen Szene bei Friedrichs eigener Familie mutet Parrs Fotografie weniger wie eine bewusste Gestaltung als vielmehr wie ein Schnappschuss an, weil zum einen das Baby anscheinend gewickelt wurde und zum anderen die Umgebung wenig einladend aussieht. 


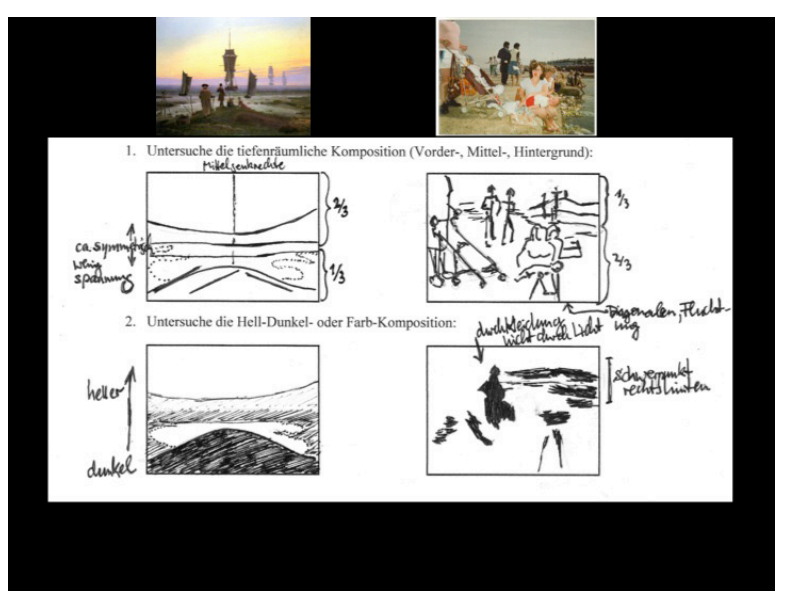

Abb. 4: Vergleichender Kompositonsvorschlag, 1.Teil

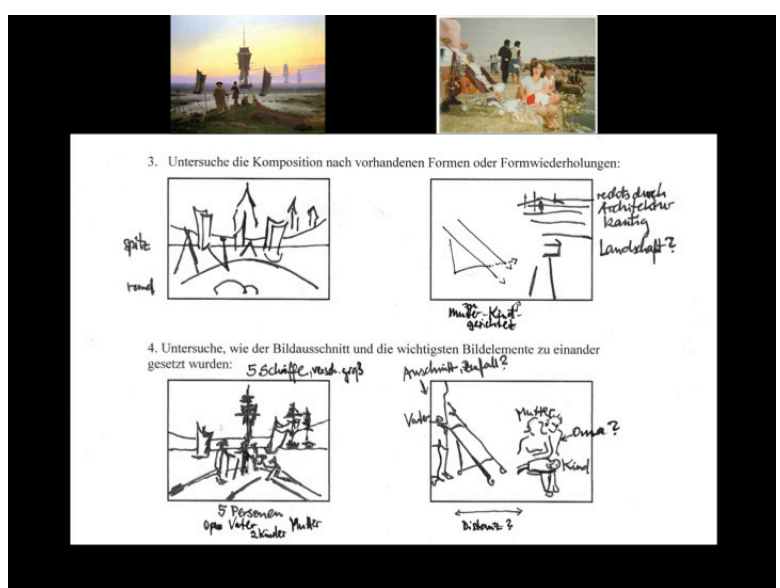

Abb. 5: Vergleichender Kompositionsvorschlag, 2.Teil

Sobald die kompositorische Untersuchung zu Wirkungen von Ruhe, Dynamik, Statik usw. gemeinsam im Unterricht erprobt wurde, kann ein weiteres Bildbeispiel eigenständig als Hausaufgabe oder bei einer Leistungsüberprüfung wie in einer Klausur in seine wesentlichen Strukturen zerlegt werden und gleichzeitig eine kulturspezifische Komponente von hierarchischen Familienkonstellationen in der Bildbetrachtung aufnehmen. Es geht um die Fotografie von Thomas Struth einer japanischen Familie (Abb. 6). Es sind sechs Personen als Familie zu sehen. Davon zwei ältere Personen, drei Personen mittleren und jüngeren Alters sowie ein Kleinkind. Es handelt sich wohl um eine Dreigenerationenfamilie. Zwischen den im Bildformat vereinzelt aufgestellten Personen sind dennoch Bindungen vorhanden. Man kann annehmen, dass die zwei ältesten Personen am rechten Bildrand als die Ältesten die Grosseltern sind. Sie bilden miteinander ein Tandem inmitten der abgebildeten Personengruppe, wobei die alte Frau im traditionellen japanischen Gewand rechts steht und der Gatte links von ihr auf einem Stein dem Betrachter zugewandt sitzt. Die Grossmutter ist mit dem Körper auf ihren Mann sowie auf die gesamte Gruppe ausgerichtet, blickt dabei aber in die Kamera. Im Bildmittelpunkt ist eine weitere Zweiergruppe. Ein Mann mittleren Alters hat im Schneidersitz, die Hände hinter dem Rücken abgestützt, zentral Platz genommen. Sein Körper ist auf die zwei älteren Personen links gerichtet, die vermutlich seine Eltern sind. Er sucht wie alle erwachsenen Personen im Bild den Betrachterblick. In seinem Schoss schlummert ein Kleinkind, das höchstwahrscheinlich ein Mädchen ist, weil es rosa Kleidung trägt. Rechts dahinter ein wenig erhöht steht ein jüngerer Mann, vielleicht der jüngere Bruder des Kindsvaters, der sich mit dem linken Fuss auf dem Stein aufstützt, auf dem Vater und Tochter ruhen. Am linken äusseren Bildrand auf der gleichen Höhe wie die Grosseltern steht eine erwachsene Frau, die demzu- 


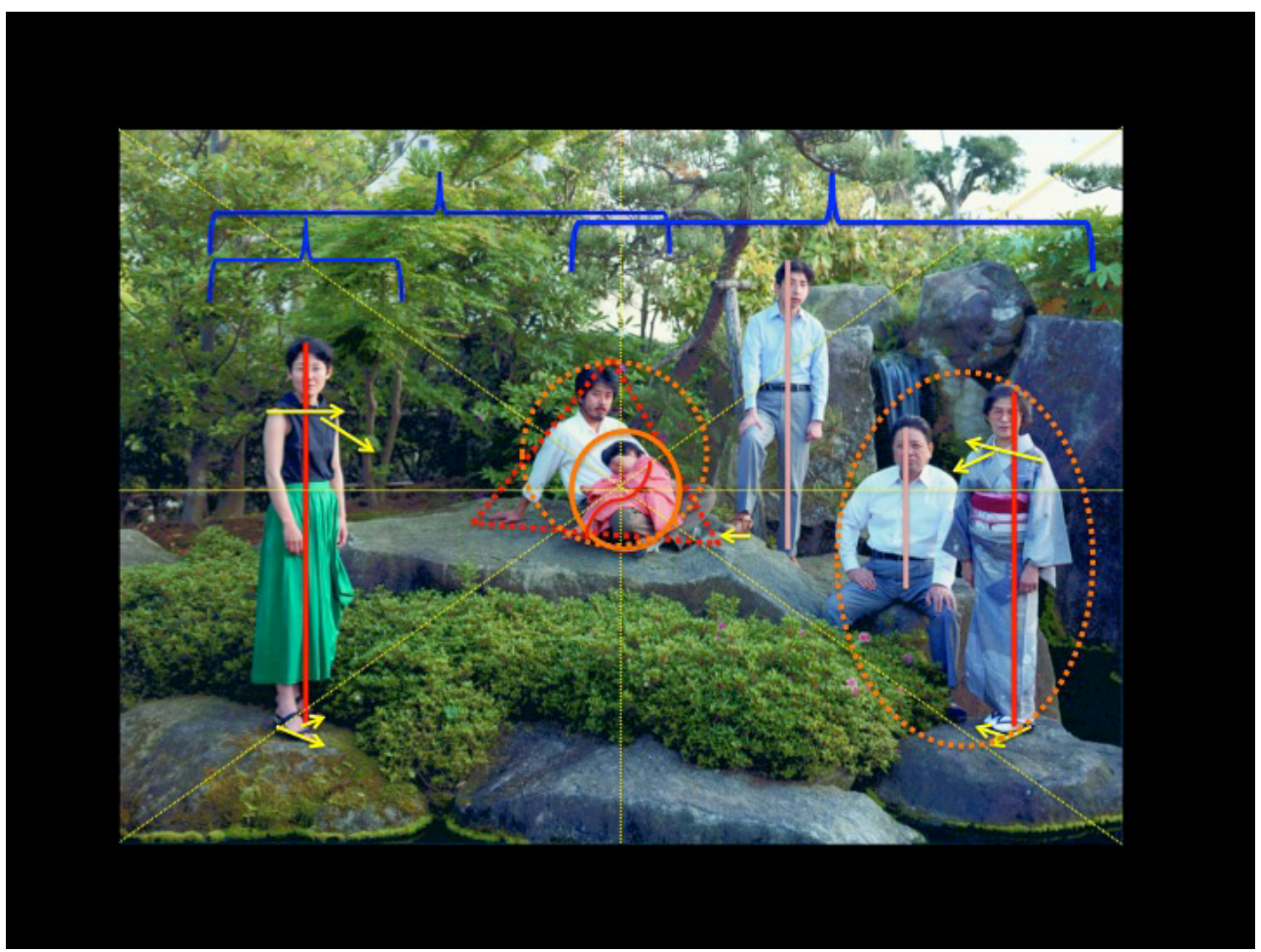

Abb. 6: Thomas Struth, Die Shimada Familie, Yamaguchi, Japan, 1986

folge die Mutter des Kleinkindes sein muss. Auch sie schaut in die Kamera, aber ihr Körper ist zur gesamten Gruppe geneigt. Obwohl also manche Personen vereinzelt im Bild stehen, bilden sie in der Ausrichtung ihrer Körper zueinander doch ein Ganzes bzw. eine Familie. Wie man es von einem Familienbild erwartet, sind alle Personen gut zu sehen, d. h. mit ihren ganzen Körpern. Gleichwohl stehen die sechs Personen nicht nah beieinander, sondern nutzen den gesamten Bildraum, um sich selbst wie auch die Familie darin zu zeigen. Das Bildformat weist in der Fläche eine harmonische Verteilung auf. Der landschaftliche Umraum unterstützt die Tiefenwirkung. Da es sich um eine asiatische respektive japanische Familie handelt, weist die Aufstellung der Einzelnen für sich sowie zueinander kulturspezifische Merkmale von gebührendem Respekt und Tradition auf. Nur das Kind im Schoss des Vaters, als noch nicht vollständig in die gesellschaftlichen Normen eingeführt, schläft bzw. darf während der Aufnahme schlafen. Es fällt ein wenig aus der Rolle. Im selben Zug drückt dieser formale Kern Geborgenheit und Schutz aus. Das einzige Kleinkind nimmt den formalen und folglich auch inhaltlichen Mittelpunkt ein. 


\section{Beratungsphasen}

Insbesondere in Verstehensprozessen gibt es unverzichtbare Lernphasen, in denen das Gespräch mit anderen zur Grundlage des Erkenntnisfortschritts wird. Im Austausch von Beobachtungen und Verstehenshypothesen, in der wechselseitigen Prüfung, im Aufbau von Argumentationen kann das Deutungsspektrum einer Bildgestaltung deutlicher erkennbar werden. (Ministerium für Schule und Weiterbildung, Wissenschaft und Forschung des Landes Nordrhein-Westfalen 1999, 27)

Vor diesen in den Richtlinien des Landes NRW verankerten Hinweisen mussten die Gruppen einen Termin als Zwischenabgabe verbindlich einhalten, zu dem erste fotografische Versuche als Ausdruck mitgebracht und zur Diskussion gestellt wurden. Die Ausdrucke wurden auf einem Tisch übersichtlich verteilt. Kriterien für ein gelungenes und weniger gelungenes Foto wurden in der Gemeinschaft entwickelt bzw. in Orientierung an der Aufgabenstellung überdacht und bei der Schlussbeurteilung wie folgt betrachtet:

- Konnten Sie die formalen Vorgaben möglichst genau einhalten, z. B. Thema, Rollenaufteilung, Form-Inhalt-Gefüge, v. a. kompositionelle Entscheidungen, Ausstattung?

- Ist der Arbeitsprozess strukturiert geplant und umgesetzt worden? (ggf. nachvollziehbare Brüche)

- Konnten Sie beratungsoffen Gestaltungsmodifikationen adäquat abwägen und vornehmen?

- Sind das Thema und die Umsetzung für einen Fremdbetrachter potenziell erkennbar, treten aber nicht plakativ auf?

- Sind die Zwischentermine mit entsprechendem Korrekturmaterial sowie der Abgabetermin eingehalten worden?

Insgesamt ist das Arrangement mit einer beruflichen Situation als Journalist, ArtDirector oder Fotograf in einer Agentur vergleichbar, wo der Auftraggeber/Kunde (hier stellvertretend die Lehrpersonen) mit bestimmten Vorgaben (siehe die gestalterische Aufgabe) adäquate Gestaltungslösungen (Endprodukte) vom Beauftragten/den Mitarbeitern/-innen (hier die Schülergruppen) erwartet. Im Berufsalltag könnte das bedeuten, dass der Auftrag an eine andere Agentur vergeben wird, wenn innerhalb des gesteckten Rahmens nicht eine zügige kreative Problemlösung als Gestaltungslösung gefunden würde. Die Vorbereitung von Handlungsfähigkeit für potenzielle Berufs- und Studienfelder ist u. a. ein Vermittlungsziel in der schulischen Bildung, vertreten von den jeweiligen Fachdisziplinen. 


\section{Zu den Schülerarbeiten}

Die hier gezeigten bildnerischen Arbeitsergebnisse dürfen nicht isoliert, sondern sollten vielmehr inmitten einer komplexen Unterrichtsreihe mit ihrem spezifisch gewachsenen Lehr-Lern-Verhältnis gedacht werden. Es wurden zu den in diesem Beitrag genannten Beispielen darüber hinaus flankierende Bildimpulse von Gruppenbildnissen aus der Malerei (z. B. Herrschafts-/Königsfamilien, Heilige Familie) und der Fotografie rezeptiv-reflexiv aufeinander bezogen, worauf die Schüler/innen mit dem eigenen Gestaltungsvorhaben teilweise reagiert haben mögen.

Es enstanden insgesamt sieben Gruppenporträts, wobei zwei Gruppen sogar nach dem «Bild-im-Bild»-Typus weitere Familienporträts aufgenommen haben, die z. B. als Zeit-Dokument ihrer inszenierten Familie im Hintergrund dienen sollen (Kindergeburtstagsfoto Abb. 14 in Abb. 13, Hochzeitsfoto in Abb. 7). Alternativ dazu wurden auch Kinderfotografien aus dem privaten Bestand in die Endkomposition integriert (vgl. Abb. 10, Abb. 12). Zwei Schülerinnen entschieden sich dafür, ihre eigene Familie für die Mitwirkung an der gestalterischen Aufgabe des Kunstunterrichts zu gewinnen (Abb. 8, Abb. 9). In der Gesamtschau der Ergebnisse fällt im Besonderen auf, dass sich die Schüler mehrheitlich für prekäre oder als schwierig empfundene Stimmungen und Konstellationen in den Familien entschieden, wobei das soziale Milieu durchaus unterschiedlich von gut-bürgerlich bis konservativ-gehoben gezielt gesucht wurde. Lediglich in einem Bild wird die Familie als harmonische und im wahrsten Sinne des Wortes miteinander verwobene Einheit dargestellt, wofür die Schülerin eine eigenwillige formale Lösung fand (Abb. 9).

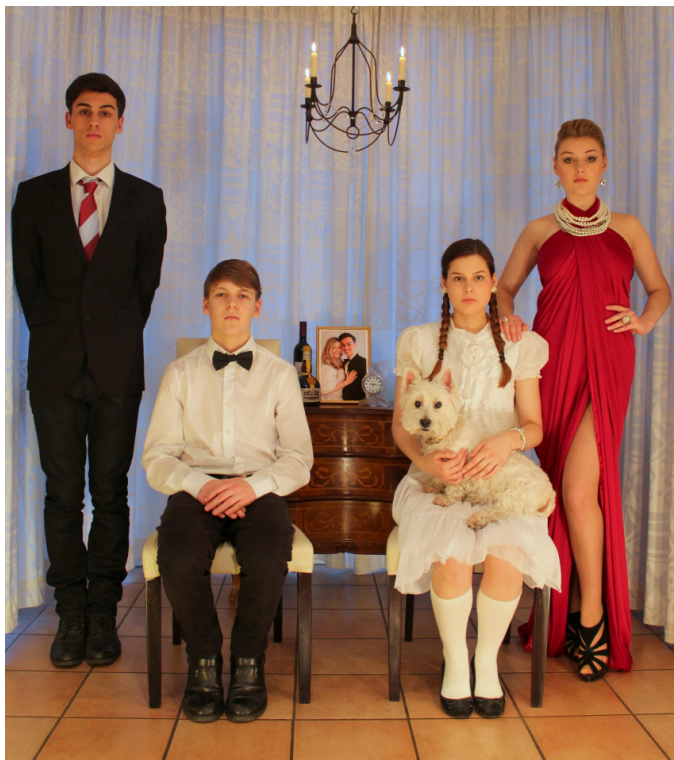

Abb. 7: Schülerarbeit Alina, Amelie, Simon und Komparse(n)

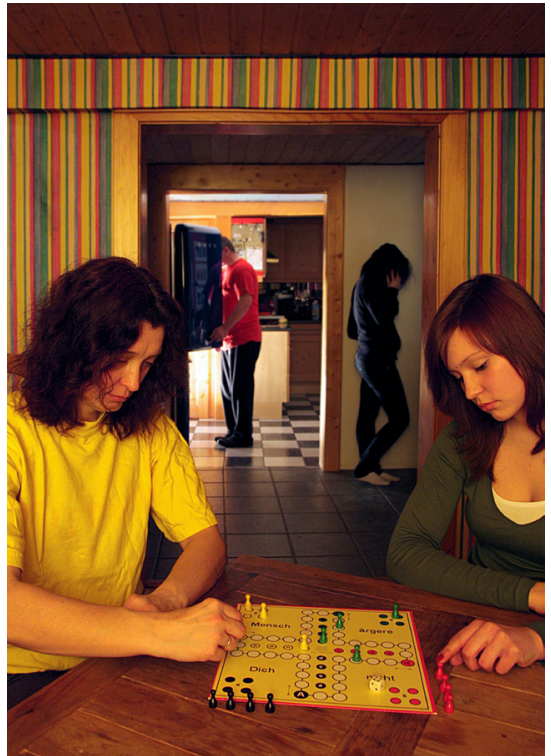

Abb. 8: Schülerarbeit Finja und die eigene Familie 


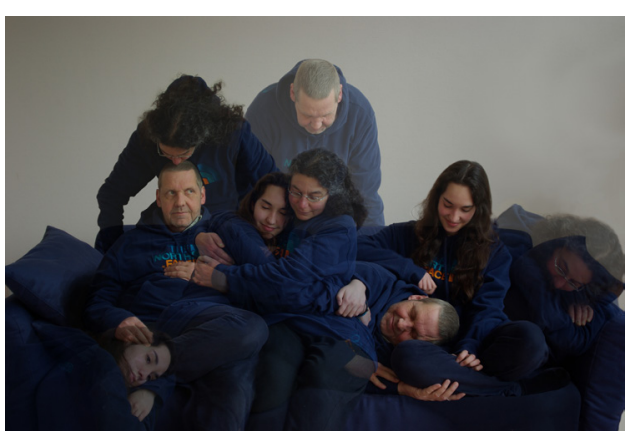

Abb. 9: Schülerarbeit Michelle B. und die eigene Familie

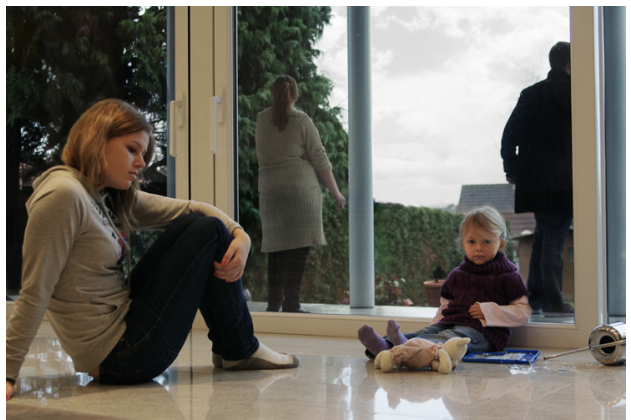

Abb. 11: Schülerarbeit Dorian, Jana, Nicole und Komparsin

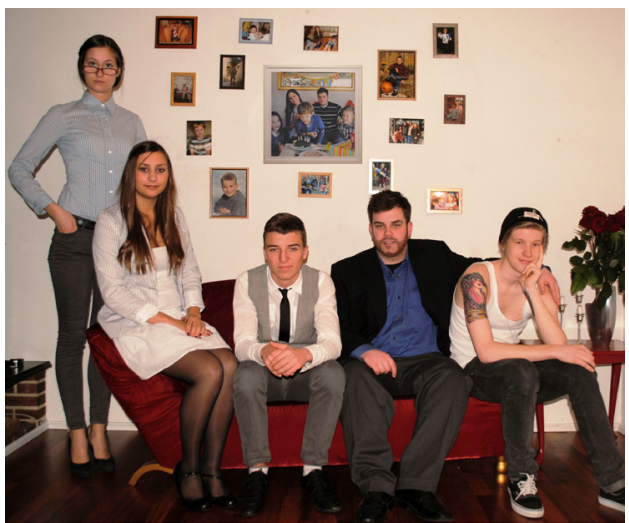

Abb. 13: Schülerarbeit David, Fabio, Inga, Lynn und Yannick (Komparsen im Hintergrundfoto)

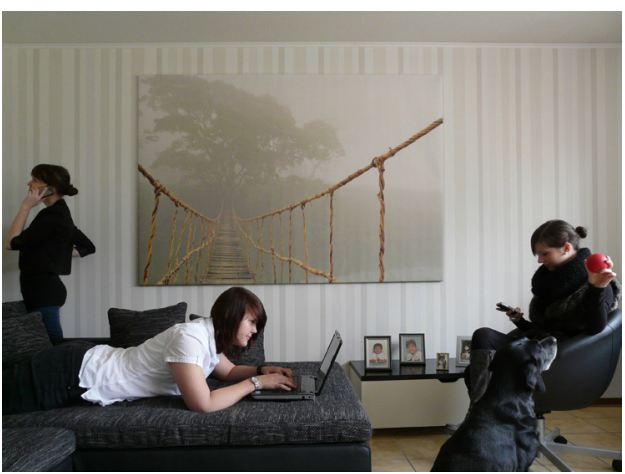

Abb. 10: Schülerarbeit Janine, Michelle St. und Komparse(n)

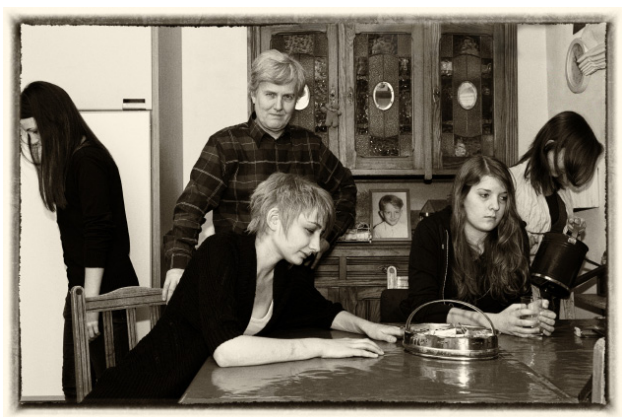

Abb. 12: Schülerarbeit Anna, Chiara, Jacqueline, Marie und Komparse (in diesem Fall: Vater von Anna)

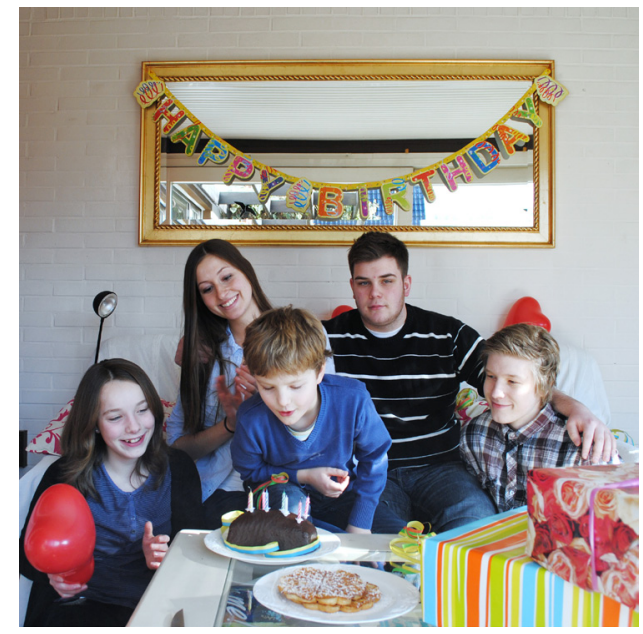

Abb. 14: Kindergeburtstagsfoto, Ausschnitt aus Abb. 13 


\section{Literatur}

Bamford, Anne. O.A. «The Visual Literacy White Paper.» http://www.adobe.com/ education/resources/k12/whitepapers/, 11.12.2010.

Bering, Kunibert. 2010. «Kunstpädagogik und Bildkultur.» In Orientierung: Kunstpädagogik. Bundeskongress der Kunstpädagogik 22.-25. Oktober, hrsg. v. Bering, Kunibert, Clemens Höxter u. Rolf Niehoff, 283-294. Oberhausen: Athena.

Bering, Kunibert, Ulrich Heimann, Joachim Littke, Rolf Niehoff und Alarich Rooch. 2006. Kunstdidaktik. Oberhausen: Athena

Boehm, Gottfried. 1994. "Die Wiederkehr der Bilder.»In Was ist ein Bild?, hrsg. v. Gottfried Boehm, 11-38. München: Fink.

Bredow, Rafaela von. 2011. «Liebe lieber unvollkommen.» DER SPIEGEL (52): 126 135.

Götting, Gesine. 2007. «Individuelle Lernförderung.»In Handbuch Schulpsychologie: Psychologie für die Schule, hrsg. v. Fleischer, Thomas, Norbert Grewe, Bernd Jötten, Klaus Seifried u. Bernhard Sieland, 98-104. Stuttgart: W. Kohlhammer.

Hahne, Robert. 2006. Wege zur Kunst: Begriffe und Methoden im Umgang mit Bildern. Braunschweig: Bildungshaus Schulbuchverlage Westermann Schroedel Diesterweg Schöningh Winklers.

Loffredo, Anna-Maria und Damian Zimmermann. 2011. "One for all and all for all - Bericht über eine (ausser)unterrichtliche Kooperation in einem LK 13 zu fotografischen Gruppenporträts.» In Schule. Künstler. Kunstpädagogen. Erfahrungen Diskurse Impulse, hrsg. v. Bering, Kunibert u. Rolf Niehoff, 139-162. Oberhausen: Athena.

Meyer, Torsten und Andrea Sabisch, Hrsg. 2009. KunstPädagogikForschung: Aktuelle Zugänge und Perspektiven. Bielefeld: Transcript.

Ministerium für Schule und Weiterbildung, Wissenschaft und Forschung des Landes Nordrhein-Westfalen. 1999. Richtlinien und Lehrpläne für die Sekundarstufe II - Gymnasium/Gesamtschule in Nordrhein-Westfalen Kunst. Frechen: Ritterbach.

Mitchell, W. J. Thomas. 1994. Picture Theory: Essays on Verbal and Visual Representation. Chicago: University Press.

Niehoff, Rolf. 2009. «Bildung - Bild(er) - Bildkompetenz(en): Zu einem wesentlichen Bildungsbeitrag des Kunstunterrichts.» In Bildkompetenz(en): Beiträge des Kunstunterrichts zur Bildung, hrsg. v. Bering, Kunibert und Rolf Niehoff, 13-42. Oberhausen: Athena.

Schäfers, Bernd. 2003. Grundbegriffe der Soziologie. Opladen: Leske + Budrich.

Wick, Rainer. 2012. «Analysen alter Meister - Von Hölzel zu Itten.»In BildGeschichte: Facetten der Bildkompetenz, hrsg. v. Hölscher, Stefan, Rolf Niehoff u. Karina Pauls, 27-41. Oberhausen: Athena. 
www.kulturundschule.de

www.paareprojekt.de

\section{Abbildungsverzeichnis}

Abb. 1 In didaktischer Absicht bearbeitete Fotografie von Annie Leibovitz (Original siehe Abb. 2).

Abb. 2 Annie Leibovitz. George Bush und sein Kabinett im Cabinet Room, Dezember 2001. Copyright Annie Leibovitz, Dezember 2001, für Vanity Fair (http://www.vanityfair.com/politics/features/2009/02/bush-oral -history200902).

Abb. 3 Links: Caspar David Friedrich, Die Lebensstufen, um 1834; Öl auf Leinwand, 73 × 94 cm; Leipzig, Museum der Bildenden Künste. Rechts: Martin Parr, Seebad New Brighton Liverpool, 1983; Farbfotografie.

Abb. 4 Vergleichender Kompositonsvorschlag, 1.Teil.

Abb. 5 Vergleichender Kompositionsvorschlag, 2.Teil.

Abb. 6 Thomas Struth, Die Shimada Familie, Yamaguchi, Japan, 1986; Farbfotografie auf Papier, 94,5 × 133,7 cm; London, Tate.

Abb. 7 Schülerarbeit Alina, Amelie, Simon und Komparse(n).

Abb. 8 Schülerarbeit Finja und die eigene Familie.

Abb. 9 Schülerarbeit Michelle B. und die eigene Familie.

Abb. 10 Schülerarbeit Janine, Michelle St. und Komparse(n).

Abb. 11 Schülerarbeit Dorian, Jana, Nicole und Komparsin.

Abb. 12 Schülerarbeit Anna, Chiara, Jacqueline, Marie und Komparse (in diesem Fall: Vater von Anna).

Abb. 13 Schülerarbeit David, Fabio, Inga, Lynn und Yannick (Komparsen im Hintergrundfoto).

Abb. 14 Kindergeburtstagsfoto in Abb. 13. 